\title{
Massage Accelerates Brain Development and the Maturation of Visual Function
}

\author{
Andrea Guzzetta, ${ }^{1}$ Sara Baldini, ${ }^{2}$ Ada Bancale,${ }^{1}$ Laura Baroncelli, ${ }^{2}$ Francesca Ciucci, ${ }^{2}$ Paolo Ghirri, ${ }^{6}$ Elena Putignano, ${ }^{2}$ \\ Alessandro Sale, ${ }^{2}$ Alessandro Viegi, ${ }^{2}$ Nicoletta Berardi, ${ }^{3,4}$ Antonio Boldrini, ${ }^{5}$ Giovanni Cioni, ${ }^{1,7}$ and Lamberto Maffei ${ }^{2,3}$ \\ ${ }^{1}$ Department of Developmental Neuroscience, Istituto di Ricovero e Cura a Carattere Scientifico Stella Maris, I-56128 Calambrone, Pisa, Italy, ${ }^{2}$ Scuola \\ Normale Superiore, I-56126 Pisa, Italy, ${ }^{3}$ Institute of Neuroscience of the Consiglio Nazionale delle Ricerche, I-56100 Pisa, Italy, ${ }^{4}$ Department of Psychology, \\ Florence University, I-50121 Florence, Italy, ${ }^{5}$ Division of Neonatology, University of Pisa, I-56100 Pisa, Italy, ${ }^{6}$ Neonatology Unit, Section of Endocrinology \\ and Dysmorphology, Azienda Ospedaliera Universitaria Pisa, I-56100 Pisa, Italy, and 7Division of Child Neurology and Psychiatry, University of Pisa, I- \\ 56128 Calambrone, Pisa, Italy
}

Environmental enrichment (EE) was shown recently to accelerate brain development in rodents. Increased levels of maternal care, and particularly tactile stimulation through licking and grooming, may represent a key component in the early phases of EE. We hypothesized that enriching the environment in terms of body massage may thus accelerate brain development in infants. We explored the effects of body massage in preterm infants and found that massage accelerates the maturation of electroencephalographic activity and of visual function, in particular visual acuity. In massaged infants, we found higher levels of blood IGF-1. Massage accelerated the maturation of visual function also in rat pups and increased the level of IGF-1 in the cortex. Antagonizing IGF-1 action by means of systemic injections of the IGF-1 antagonist JB1 blocked the effects of massage in rat pups. These results demonstrate that massage has an influence on brain development and in particular on visual development and suggest that its effects are mediated by specific endogenous factors such as IGF-1.

\section{Introduction}

Environmental enrichment is a widely used paradigm for investigating the influence of sensory experience on brain and behavior. Enriched environment (EE) was first defined by Rosenzweig et al. (1978) as "a combination of complex inanimate and social stimulation." "Enriched" animals are reared in larger cages and in larger groups, and a variety of toys, tunnels, nesting material, and stairs are present and changed frequently. In addition, animals are typically given the opportunity for voluntary physical activity on running wheels. EE has remarkable effects on adult brain function in several species (van Praag et al., 2000; Nithianantharajah and Hannan, 2006; Sale et al., 2007). Recently, it has been shown that EE from birth leads to a conspicuous acceleration of visual system development in rodents, appreciable at the behavioral, electrophysiological, and molecular level (Cancedda et al., 2004; Landi et al., 2007).

It has been suggested that, in the earliest phases of exposure to $\mathrm{EE}$, the effects of EE do not stem from a direct action on the developing pups but rather from a variation in maternal behavior in enriched conditions (Cancedda et al., 2004; Sale et al., 2004). Indeed, it has been shown that pups in EE receive a higher level of

Received Nov. 19, 2008; revised Feb. 25, 2009; accepted Feb. 28, 2009.

This work was supported by Ministero dell'Istruzione, dell'Università, e della Ricerca (Progetti di Rilevante Interesse Nazionale) (L.M.) and Fondazione Mariani (G.C.). We thank Dr. Graziano Tinivella and Dr. Luc Zyw for their help with the statistical analysis. Technical help from Giulio Cappagli is gratefully acknowledged.

Correspondence should be addressed to Prof. Lamberto Maffei, Institute of Neuroscience of the Consiglio Nazionale delle Ricerche, Via G. Moruzzi, 1, 56100 Pisa, Italy. E-mail: maffei@in.cnr.it.

DOI:10.1523/JNEUROSCI.5548-08.2009

Copyright $\odot 2009$ Society for Neuroscience $\quad$ 0270-6474/09/296042-10\$15.00/0 tactile stimulation through licking, grooming, and physical contact (Sale et al., 2004). The level of licking and grooming provided by the mother has been shown to influence hippocampal structure and function, to affect molecules crucial for plasticity such as BDNF and NMDA receptors, and to leave long-lasting traces in the offsprings' behavior. The offsprings of mother rats with high or low licking and grooming behavior show, once adult, differences in novelty reaction, in exploratory behavior, in spatial learning and memory, in the level of glucocorticoids receptors in the hippocampus, leading to a difference in the feedback control on hypothalamus-pituitary-adrenal axis, and in spine density and synaptic plasticity in the hippocampus (Liu et al., 2000; Weaver et al., 2004, 2006, 2007; Meaney and Szyf, 2005; Champagne and Curley, 2009). Studies in rodents devoted to the exploration of a specific role of tactile stimulation have shown that the negative effects produced by maternal separation/deprivation or prenatal stress on pup growth, growth hormone $(\mathrm{GH})$ secretion, HPA axis, and BDNF and synaptophysin expression, were rescued by tactile stimulation of the pups (Schanberg and Field, 1987; Burton et al., 2007; Chatterjee et al., 2007). Working in preterm infants, Schanberg and Field (1987) found evidence that massage promoted a faster weight gain and a lower level of cortisol in massaged infants.

Recently, body massage and multisensory stimulation are increasingly included in neonatal care in human newborns, with some evidence for a significant effect on the rate of weight gain (Vickers et al., 2004). Neither in animals nor in humans it is known, however, whether massage may affect brain development.

To address this issue, we explored the effects of massage ther- 
apy on brain development and in particular on visual system development in a group of healthy preterm infants (gestational age between 30 and 33 weeks) and, in parallel, in early postnatal rat pups. We found that massage influences the maturation of visual evoked potentials (VEPs) and of visual acuity both in human infants and in rat pups. In infants, we also investigated the maturation of electroencephalographic (EEG) activity, finding a clear acceleration in massaged infants.

We have found recently that IGF-1 is a key factor mediating EE effects on visual cortical development (Ciucci et al., 2007): EE increases the number of IGF-1-positive neurons in the visual cortex; increasing IGF-1 in the visual cortex of non-EE rats by means of osmotic minipumps mimics EE effects, accelerating visual acuity development while blocking IGF-1 action in the visual cortex of EE rats by means of the IGF-1 receptor antagonist JB1, blocks EE action on visual acuity development. We investigated whether IGF-1 could be involved in mediating the effects of massage on visual development. We found that massage led to increased levels of blood IGF-1 and IGF1BP3 in human infants and to an increased number of IGF-1-positive neurons in the cortex in rat pups. Antagonizing IGF-1 action blocked the effects of massage in rat pups.

\section{Materials and Methods \\ Massage in human infants}

Ten newborns with a gestational age between 30 and 33 weeks, admitted to the Neonatal Intensive Care Unit (NICU) of the University Hospital of Pisa, were selected for the study on the basis of very precise conditions, to exclude confounding factors. Inclusion criteria were a birth weight between the $25^{\circ}$ and the $75^{\circ}$ centile, a birth length $>10^{\circ}$ centile, no abnormalities on brain ultrasound, and parents' approval. Infants with genetic anomalies, congenital heart malformations, CNS dysfunction, as indicated by neurological examination, or medical conditions primarily related to immaturity, such as respiratory distress syndrome, hyaline membrane disease, apnoea, elevated bilirubin, and mild hypoglycemia and hypocalcemia, were excluded. Ten newborns matched for age, sex, and birth weight were selected according to the same criteria as controls. Written informed parental consent was obtained in all cases.

\section{Massage therapy}

Massage therapy was begun on day $10( \pm 1)$. Sessions were performed three times a day for two blocks of $5 \mathrm{~d}$ each, separated by a $2 \mathrm{~d}$ interval. The massage was always performed by the same person, expert in this technique. Each massage session was performed $\sim 60$ min before feeding and at least $2 \mathrm{~h}$ after the completion of the previous stimulation. Each treatment session consisted of 10 min of tactile stimulation, followed by 5 min of kinesthetic stimulation. During tactile stimulation, the infant was placed prone and was given moderate pressure stroking with the flats of the fingers of both hands (supplemental Figs. 1, 2, available at www. jneurosci.org as supplemental material). Head, neck, shoulders, buttocks, and both legs and arms were stimulated. For the kinesthetic phase, the infant was placed in a supine position. Passive flexion/extension movements of the limbs in sequence were applied (supplemental Fig. 3, available at www.jneurosci.org as supplemental material). During massage, a soft tune of classical music (Johannes Brahms' Wiegenlied: Guten Abend, gute Nacht, Opus 49, Number 4) was played in the environment. In the NICU in Pisa, parents are admitted to the ward for three to four times per day, for $\sim 45$ min each. Massage was performed when the mother was not present, between two feeding times; during massage, nurses were asked not to perform routine care interventions.

Infants in the massaged and control group received identical medical nursing care, with the exception of the massage. In the NICU in Pisa, routine care includes a number of interventions designed to minimize the stress of the infant in the neonatal intensive care environment, by reducing noise and light, minimal handling, longer rest periods, and gentle individualized stimulations (Als et al., 1994; Symington and Pinelli, 2006). All infants in the NICU, including massaged and control infants included in this study, were exposed to classical music diffused in the environment during day time. There was no difference in the time spent with the mothers between the massaged and control groups.

\section{Premassage and postmassage battery}

A battery of exams was performed at $\sim 1$ week of age ( $\pm 1 \mathrm{~d})$ (T1) and at $\sim 4$ weeks of age ( $\pm 2 \mathrm{~d}$ ) (T2) which, for infants in the massage group, corresponds to before and after the massage therapy. Battery consisted of the following exams: (1) clinical assessment (body weight, length, and head circumference); (2) electrophysiological assessment [EEG, flash VEPs (fVEP), brainstem auditory-evoked potentials (BAEPs)]; and (3) serum examinations [IGF1, IGF binding protein-3 (IGFBP3), glucose, insulin, cortisol, and thyroid hormones]. Clinical assessment and blood drawing were performed between 8:00 and 9:00 A.M., whereas the eletrophysiological assessment was performed between 2:00 and 6:00 P.M., in the same day.

The amount of weight gain between T1 and T2 was not significantly different between massaged and control subjects ( $t$ test, $p=0.52$; mean \pm SEM weight gain, $561 \pm 83.5 \mathrm{~g}$ in massaged infants, $535 \pm 83 \mathrm{~g}$ in control infants).

\section{Electrophysiological measures}

All electrophysiological exams were obtained at the bedside using a portable digital EEG system (Brain Quick; Micromed), by an expert technician, uninformed of the subject belonging to massaged or control group.

EEG. The EEG recordings were obtained from eight active electrodes (Fp1-Fp2, C3-C4, O1-O2, T3-T4 of the International 10-20 System) applied to the scalp using adhesive paste. From each tracing, $30 \mathrm{~min}$ of artifact-free recording was analyzed and the following parameters were evaluated (Biagioni et al., 1994, 1996): (1) for maximum interval duration (seconds), an interval was taken as a period of low activity $(<30 \mu \mathrm{V})$ in all leads; (2) for minimum burst duration (seconds), a burst was defined as waves emerging from the low activity of the interval in at least half of the leads (waves $>30 \mu \mathrm{V}$ observed in less than half of the leads were considered to interrupt the interval but were not taken to be bursts), and the beginning of the first wave and the end of the last one were taken as the onset and the offset of the burst; (3) for incidence of $8-20 \mathrm{~Hz}$, activities superimposed on $\delta$ waves (the $\delta$ brush pattern): they were only considered present on a burst when reaching at least one-half of their maximum amplitude; and (4) incidence of temporal sawtooth (i.e., a rhythmic regular 4-7 Hz activity, detectable on temporal regions).

Flash VEPs. The active electrode was placed at $\mathrm{Oz}$, the reference electrode at $\mathrm{Fz}$, and the ground electrode at $\mathrm{Cz}$, according to the International 10-20 System. The impedances were usually below $5 \mathrm{k} \Omega$. Flash light stimuli using light-emitting diodes (light-emitting diode goggles) were presented $\sim 3-5 \mathrm{~cm}$ in front of the infant's eyes at a frequency of 0.2 $\mathrm{Hz}$ with duration of $10 \mathrm{~ms}$. One hundred responses were averaged for each trial with a bandpass of $1-100 \mathrm{~Hz}$ and a sweep time of $1 \mathrm{~s}$. Two or more trials were recorded to ensure reproducibility of the waveform. Responses with excessive artifact (more than $\pm 200 \mu \mathrm{V}$ ) were automatically rejected. We analyzed the waveform of negative peaks with a mean latency of $\sim 300 \mathrm{~ms}$ (N300) and measured the exact latency in each case.

$B A E P s$. Brainstem auditory-evoked potentials were recorded with disk electrodes placed, according to the International 10-20 System, on the vertex (active electrode) and the mastoid (reference electrode) ipsilateral to the stimulation. The contralateral ear was masked by white noise of 40 $\mathrm{dB}$ during stimulation. Each ear was stimulated with a series of squarewave rarefaction clicks through headphones at $90 \mathrm{~dB}$. The repetition rate of stimuli was $15 \mathrm{~Hz}$ (15/s), the recording window was $10 \mathrm{~ms}$ from click onset, and each trial consisted of 2000 artifact-free clicks. Absolute latency and amplitude for waves I-V and interpeak latencies I-III, III-V, and I-V were measured. The latency and amplitude values obtained for the left and right ears were averaged to represent each case by one value in statistical analysis.

All electrophysiological recordings were assessed offline, by an expert examiner, unaware of whether a tracing belonged to massaged or control subject. 


\section{Hormone assays}

Blood samples were obtained between 8:00 and 9:00 A.M. from fasting subjects, by a 23-gauge butterfly needle connected to an injection syringe, transferred to a test tube without anticoagulant, centrifuged at room temperature, and stored at $-20^{\circ} \mathrm{C}$. Samples thawed just before the assays that were performed within 3 months from blood collection. Serum IGFBP-3 was measured by immunoradiometric assay (IRMA) (Diagnostic Systems Laboratories). The intra-assay coefficient of variation (CV) was $1.8-3.9 \%$, the interassay CV was $0.5-1.9 \%$, and the sensitivity limit was $0.5 \mathrm{mg} / \mathrm{L}(17.5 \mathrm{nmol} / \mathrm{L})$. Serum IGF-I was measured by IRMA (Nichols Institute Diagnostics). IGF-I was measured after extraction obtained by acidification. The intra-assay CV was $3.3-4.6 \%$, the interassay CV was $9.3-15.8 \%$, and the sensitivity limit was $6 \mu \mathrm{g} / \mathrm{L}(0.8 \mathrm{nmol} / \mathrm{L})$.

Cortisol. Serum cortisol was measured by radioimmunoassay (BykSangtec Diagnostica). The intra-assay CV was $2.1-4.0 \%$, the interassay CV was 3.2-9.0\%, and the sensitivity limit was $13.8 \mathrm{~nm}$. All analyses were performed blind.

\section{Behavioral acuity}

This was assessed binocularly by the Bébé Vision Tropique acuity card procedure (Vital-Durand, 1992). This method is based on an inborn preference for a pattern (black and white gratings of different stripe widths) over a uniform field, depicted on cards with decreasing stripe widths. The location of the left/right position of the test stimulus varies randomly. An observer, blind to the effective position of the stimulus, judges the infant's reaction as to the location of the test stimulus on the basis of eye and head movement. The threshold of acuity is taken as the finest stripe width for which the subject consistently responds correctly. Acuity values were expressed in cycles per degree $\left(\mathrm{c} /{ }^{\circ}\right)$. Assessment was performed in each infant at 3, 7, 9, and 12 months corrected age by the same vision scientist, following strictly recommended procedure of acuity card testing (Teller et al., 1986; van Hof-van Duin et al., 1992; VitalDurand, 1992).

\section{Massage in rat pups}

Animals. All experiments were performed on rats in accordance with the Italian Ministry of Public Health guidelines for care and use of laboratory animals. Long-Evans hooded rats lived in an animal house at $21^{\circ} \mathrm{C}$ temperature, $12 \mathrm{~h}$ light/dark cycle, and food and water available ad libitum. Parturition was checked one time a day, and the day of birth was considered postnatal day 0 (P0). The mother and its litter were housed in $26 \times$ $42 \times 18 \mathrm{~cm}$ Plexiglas cages.

Animal groups and tactile stimulation protocol. We used three groups of rat pups. One group ("massage group") was separated from the mother three times per day (9:00 A.M., 2:00 P.M., and 7:00 P.M.) from P1 to P12 and received tactile stimulation. One group was separated from the mother for the same amount of time and at the same period of the day but did not receive any stimulation ("maternal separation group"). The other group was left undisturbed with the mother ("control group"). For the massage and maternal separation groups the procedure was the following: the mother was temporarily put in another cage, and the entire litter of pups was removed from the home cage and put in a box over a warm plate $\left(34^{\circ} \mathrm{C}\right)$ to recreate the temperature of the nest. Pups in the massage group were then individually subjected to the stimulation protocol, whereas pups in the maternal separation group were left in the box over the warm plate. At the end of each session, animals were replaced with their mother. The presence of the maternal separation group controls for the effects of removing pups from the cage and separating them from the mother.

The stimulation protocol combined gently stroking and massaging to mimic maternal care. Each animal received 5 min of tactile stimulation: 2 min with a wet soft paintbrush on their back, on the head, on the limbs, and on the abdomen to mimic licking; 1.5 min massage with finger tips on both sides of their back combined with passive gentle movement of their limbs; $1.5 \mathrm{~min}$ with a soft toothbrush on the back and the abdomen to mimic grooming.

At P12, the body weight was assessed in 108 animals, subsequently used, at later ages, for the electrophysiology, behavior, or immunohistochemistry experiments described below. We found that massage and maternal separation pups both weighed more than control pups; the weight of separated pups was also higher than that of the massaged pups [one-way ANOVA, $p<0.001 ; F=15.218$, multiple comparisons HolmSidak procedure, weight of control pups $(n=35$; mean \pm SEM, $23.8 \pm$ $0.7 \mathrm{~g}$ ) lower than massage and maternal separation pups (massage pups, $n=30$, mean \pm SEM, $26.1 \pm 0.6$ g; maternal separation pups, $n=43$, mean \pm SEM, $28.6 \pm 0.6 \mathrm{~g}$ ), and weight of massage pups lower than maternal separation pups $(p<0.05)]$.

No difference in body weight between massage and maternal separation group was found at P25; rats in both groups weighed more than rats in control group [one-way ANOVA, $F=7.39, p=0.004$, multiple comparison procedure Holm-Sidak method, weight of control rats $(n=7$; three litters; mean \pm SEM, $53 \pm 3 \mathrm{~g}$ ) lower than massage and maternal separation rats (six litters, massage, $n=11$ pups, mean \pm SEM, $63.1 \pm$ 1.7; maternal separation, $n=11$ pups, mean \pm SEM, $62 \pm 1.5)]$.

$E E$. Enriched environment consisted of large wire netting cages $(60 \times$ $50 \times 80 \mathrm{~cm}$ ) with three floors containing several food hoppers, two running wheels to allow voluntary physical activity, and differently shaped objects (tunnels, shelters, and stairs) that were completely substituted with others once a week. Every cage housed at least four to five females and their pups. Cages for standard environment (non-EE) were standard laboratory cages $(30 \times 40 \times 20 \mathrm{~cm})$ housing one dam with her pups as established by the Italian law for the care of laboratory animals.

Flash VEP recordings. Animals in the massage and control groups were anesthetized by intraperitoneal injection with avertine $(1 \mathrm{ml} / \mathrm{hg})$, and body temperature was continuously monitored and maintained at $37^{\circ} \mathrm{C}$ by a thermostated electric blanket. fVEPs were elicited using a full-field flash generated on a monitor $\left(20 \times 22 \mathrm{~cm}\right.$; luminance, $70 \mathrm{~cd} / \mathrm{m}^{2}$; flash rate, $0.3 \mathrm{~Hz}$; duration, $0.66 \mathrm{~s}$ ) positioned $20 \mathrm{~cm}$ from the rat's eyes. Recording electrodes were positioned on the scalp overlying the primary visual cortex, with the ground electrode on the front. The recorded activity was digitally bandpass filtered (direct current, $120 \mathrm{~Hz}$ ), and 50 responses were averaged. The results were displayed scalp positive up. Flash VEPs were recorded at P14-P18. For all time points, $n=4$ rats for each experimental group, coming from four litters. All recordings were performed blind.

VEP visual acuity measurements. A total of 30 rats from 10 litters was used (massage group, $n=11$ rats; maternal separation group, $n=12$ rats; control group, $n=7$ rats). Rats were anesthetized with an intraperitoneal injection of $20 \%$ urethane $(0.7 \mathrm{ml} / \mathrm{hg}$; Sigma $)$ and mounted on a stereotaxical apparatus allowing full viewing of the visual stimulus. Additional doses of urethane $(0.03-0.05 \mathrm{ml} / \mathrm{hg}$ ) were used to keep anesthesia level stable throughout the experiment. During electrophysiology, the body temperature of rats was monitored with a rectal probe and maintained at $37.0^{\circ} \mathrm{C}$ with a heating pad. A glass micropipette $(2-2.5 \mathrm{M} \Omega)$ was inserted into the binocular primary visual cortex in correspondence with the vertical meridian representation (Oc1B). Electrical signals were amplified, bandpass filtered $(0.1-120 \mathrm{~Hz})$, and averaged (at least 50 events in blocks of 10 events each) in synchrony with the stimulus contrast reversal. Transient VEPs in response to abrupt contrast reversal $(0.5-1 \mathrm{~Hz})$ of a sinusoidal grating were evaluated in the time domain by measuring the peak-to-baseline amplitude and peak latency of the major component. VEPs in response to a blank field were also frequently recorded to have an estimate of the noise. For each animal, VEP amplitude was plotted as a function of log spatial frequency, and visual acuity was determined by linearly extrapolating VEP amplitude to $0 \mathrm{~V}$. All recordings were performed blind.

Behavioral measure of visual acuity. A total of 39 rats from 12 litters was used (control group, $n=13$ rats; massage group, $n=15$ rats; maternal separation group, $n=11$ rats). Visual acuity was measured using a twoalternative forced-choice procedure (Prusky et al., 2000). Two monitors $(31 \times 22 \mathrm{~cm}$ in size $)$ were placed side by side at the wide end of trapezoidal-shaped tank containing $22^{\circ} \mathrm{C}$ water. A vertically oriented square-wave grating ( $90 \%$ contrast) was displayed randomly on one of the two monitors, whereas the other displayed uniform gray of the same mean luminance. A reward platform was hidden under the surface of the water directly below the monitor displaying the grating. Rats were trained to swim from the release point, located at the end of the tank, to the grating side, in which they found the hidden platform. A short barrier 
$(50 \mathrm{~cm})$ was placed in the tank between the stimuli, creating a choice plane. The location of the grating on any trial was pseudorandomized. When performance with a low spatial frequency $\left(0.117 \mathrm{c} /{ }^{\circ}\right)$ reached at least $80 \%$ correct discrimination for two consecutive sessions, the training phase ended. To measure visual acuity, the limit of the discrimination was estimated by increasing the spatial frequency of the grating until performance fell below 70\% accuracy. For each rat, several sessions of visual acuity estimate were run and a frequency of seeing curve was constructed from the total data $(90-110$ trials at least). The spatial frequency corresponding to $70 \%$ accuracy was taken as the acuity value. All measurements were performed blind.

JB1 injections. A total of 14 rats from four litters was used. Injections of JB1, an IGF-1 receptor antagonist (Fernandez et al., 1999; Ciucci et al., 2007), were performed subcutaneously in massaged pups ( $n=8$, massage plus JB1 group) from P1 to P9. For each pup, a single injection per day was performed, at 9:00 A.M. JB1 concentration was $50 \mathrm{ng} / \mu \mathrm{l}$, and the volume of the injection was adjusted to the pup weight to reach a dose of $18 \mathrm{ng} / \mathrm{g}$ body weight. Six massaged pups were treated with subcutaneous injections of saline (massage plus saline group), as a control for the effects of subcutaneous injections in massaged rats.

Immunohistochemistry. A total of 48 rats from 10 litters was used (massage group, $n=16$ rats; maternal separation group, $n=16$ rats; control group, $n=16$ rats) Long-Evans hooded rats aged between P14 and P25 were used ( $\mathrm{P} 15$, massage group, $n=4$; maternal separation group, $n=4$; control group, $n=4$; P18, massage group, $n=4$; maternal separation group, $n=4$; control group, $n=4$; P25, massage group, $n=4$; maternal separation group, $n=4$; control group, $n=4$ ). Protocol was as in the study of Ciucci et al. (2007). Briefly, $35 \mu \mathrm{m}$ coronal sections were cut on a microtome. Polyclonal anti-IGF-1 (1:500) antibody was revealed with biotinylated secondary antibody goat anti-rabbit $\operatorname{IgG}(1: 200)$, followed by fluorescein-conjugated extravidin (1:300). For neuronal-specific nuclear protein $(\mathrm{NeuN})$, we used a monoclonal antibody (1:500) revealed with Alexa 568 (1:400). At all ages, images were acquired with a confocal Olympus microscope at $20 \times$ magnification (numerical aperture, 0.7; field, $707 \times 707 \mu \mathrm{m}$ acquired at $1024 \times 1024$ pixels) to analyze the number of IGF-1- and NeuN-positive cells in sections double labeled for IGF-1 and NeuN. For each animal, at least three Oc1B sections and three Tel sections were analyzed. Counts were done on the entire thickness of Oc1B and Te1. The number of IGF-1-positive cells was normalized to the number of NeuN-positive cells. All analyses were performed blind.

Plasma corticosterone. Corticosterone was measured by means of EIA kit from Chematil according to the provided protocol. Briefly, whole blood samples were collected from 18 rat pups from four litters at P14 (massage group, $n=6$ pups; maternal separation group, $n=6$ pups; control group, $n=6$ pups) by decapitation between 9:00 and 10:00 A.M. and allowed to settle on ice. Resulting samples were centrifuged (6000 $\mathrm{rpm}, 10 \mathrm{~min}, 4^{\circ} \mathrm{C}$ ), and supernatants were stored at $-80^{\circ} \mathrm{C}$ until use. Twenty-five microliters of each sample were run in duplicate on polystyrene microtiters plate with goat anti-rabbit IgG immobilized to the inside wall of each well. One hundred microliter of rat corticosterone antiserum were added to each well, and the plate was shaken for $60 \mathrm{~min}$ at room temperature. After extensive washing, $100 \mu \mathrm{l}$ of tetramethylbenzidine solution were added and incubated for $15 \mathrm{~min}$. The reaction was stopped with an additional $100 \mu \mathrm{l}$ of stopping solution, and the plate was read with a microplate reader at $450 \mathrm{~nm}$. Corticosterone amount was established by means of a calibration curve reporting absorbance at $450 \mathrm{~nm}$ on abscissa versus log [standard concentration].

Statistical analysis. Statistical analysis was performed with SigmaStat; parametric or nonparametric analysis was performed according to the results of the test for normality of the data. Significance level was 0.05 . Effect size was calculated as Cohens's $d$ and the difference between the two means divided by pooled SD for the data.

\section{Results}

EEG maturation is accelerated in massaged preterm infants

During the first weeks after delivery, EEG activity in preterm infants undergoes rapid and remarkable changes, which are a robust index of the maturational stage of the brain (StockardPope et al., 1992). The most conspicuous change in the EEG is the transition from discontinuous to continuous activity, with a progressive reduction of the duration of the intervals between bursts of activity (Stockard-Pope et al., 1992; Scher et al., 1994). We have measured the maximum length of interburst intervals in the EEG during quiet sleep before and after the cycle of treatment [first assessment, 7-9 d after birth (T1); second assessment, 3 weeks later (T2)]: as evident in Figure $1 a$, there is a much larger degree of shortening of the interburst intervals between the two assessments in massaged infants with respect to controls; the median reduction is $7 \mathrm{~s}$ [interquartile (IQ) $=5.3-9$ ] in massaged infants and only $2.8 \mathrm{~s}(\mathrm{IQ}=2-3.8)$ in controls (Mann-Whitney $U$ two independent sample test, $p=0.011 ; n=10$ for both groups; Cohen's $d=1.33$ ).

\section{Maturation of the visual system is accelerated in massaged preterm infants}

As an index of early visual system maturation, we have used the latency of fVEPs. At this age, the most prominent peak of fVEP is N300, the latency of which exhibits a progressive shortening during the first months of life (Tsuneishi et al., 1995). We assessed N300 latency before and after the cycle of treatment (T1 and T2), finding a significantly larger reduction in the treated infants than in the control group (Fig. 1b); in the latter, the median reduction in N300 latency during the 3 weeks interval between the first and second assessment is $10.8 \mathrm{~ms}$ (IQ $=4-24.2$ ), whereas in the massage group, the median reduction is $42.8 \mathrm{~ms}$ (IQ $=25.1-$ 63.5) (difference in median reduction in N300 latency in massage vs control significant, Mann-Whitney test, $p=0.013 ; n=10$ in both groups; Cohen's $d=1.40$ ). This supports a role for massage therapy in the enhancement of fVEP maturation. An acceleration was not observed for the maturation of the latencies and interpeak intervals of signals evoked from totally subcortical structures, such as brainstem-auditory evoked potentials [ (mean interpeak interval $I-V$ reduction between $\mathrm{T} 1$ and $\mathrm{T} 2$ (mean \pm SEM), $0.268 \pm 0.08 \mathrm{~s}$ for massage group, $n=10 ; 0.482 \pm 0.24 \mathrm{~s}$ for control group, $n=10$, difference not significant, $t$ test, $p=$ 0.374; mean reduction in peak III latency (mean \pm SEM), $0.13 \pm$ 0.09 s for massage group, $n=10 ; 0.37 \pm 0.10 \mathrm{~s}$ for control group, $n=10$, difference not significant $t$ test, $p=0.102$; mean reduction in peak V latency (mean \pm SEM), $0.31 \pm 0.09 \mathrm{~s}$ for massage group, $n=10 ; 0.61 \pm 0.14$ s for control group, $n=10$, difference not significant $t$ test, $p=0.1)$.

To investigate whether our protocol of stimulation produced an effect on visual development that outlasted the end of the treatment, we measured behavioral visual acuity at 3 months of age by means of a two-alternative forced-choice preferential looking. Visual acuity is a robust index of maturation of the visual system. Visual acuity normally shows a sharp increase during the first $6 / 8$ months of life and then slowly reaches adult values at $\sim 36 / 48$ months (Vital-Durand, 1992). At 3 months post-term age, visual acuity was significantly better in massaged infants than in controls ( $($ Fig. 1c) $(t$ test, difference in visual acuity between massage and control infants, $p<0.001$, Cohen's $d=2.2, n=10$ for both groups; mean \pm SEM visual acuity, $5.7 \pm 0.2 \mathrm{c} /{ }^{\circ}$ in massaged infants, $4 \pm 0.3 \mathrm{c} /{ }^{\circ}$ in control infants); the difference is $\sim 45 \%$.

Altogether, these findings suggest that massage therapy is effective in accelerating brain maturation in preterm infants, and, in particular, the maturation of visual function. It is of great interest that, in our group of preterm infants, two monozygotic twins were included, assigned to different arms of the study. Their results confirmed on a single case-control basis the general pattern of results obtained for the entire group (Table 1). 
The effects of massage therapy were not permanent, because differences between massaged and control infants can be no longer detected at 7 months (Fig. 1d) (Mann-Whitney test, $p=0.79 ; n=8$ for both groups) and 12 months (data not shown). This is consistent with the animal model, in which the effects of EE on the acceleration of visual maturation were only detected in the first part of the developmental curve (Cancedda et al., 2004; Landi et al., 2007).

\section{Massage increases blood IGF-1 and IGFBP3 and reduces cortisol levels in preterm infants}

It has been shown that massage in rat pups and preterm infants causes an increase in GH production (Schanberg and Field, 1987). GH controls IGF-1 production, and indeed a very recent study found an increase in serum IGF-1 associated with massage in very preterm infants (Field et al., 2008). A molecule of great relevance for development, IGF-1 has been shown to be involved in visual cortical experiencedependent plasticity (Tropea et al., 2006) and to mediate EE effects on visual acuity development in rodents (Ciucci et al., 2007). In addition, very recent papers have shown that IGF-1 and a molecule of the IGF-1 pathway, IGFBP3 could be protective against proliferative retinopathy of prematurity (ROP), a severe and relatively frequent visual disorder in preterm infants (Löfqvist et al., 2006, 2007). It was therefore very important to explore the IGF-1 pathway in our preterm infants both to gain insight on the possible mediators of the effects of the massage therapy on brain development and in view of potential clinical applications of the therapy.

We have measured blood IGF-1 and IGFBP-3 immediately before and after the period of treatment. We found that IGF-1 levels decrease between the first and the second assessment in both massaged and control infants, but this decrease (Fig. 1d) is much smaller in massaged infants than in control infants $[n=7$ for both groups, two-way repeated measures ANOVA, treatment $(\mathrm{df}=1) \times$ time $(\mathrm{df}=1)$, pairwise multiple comparison procedure Holm-Sidak, factor time within massage, IGF-1 levels at T1 vs T2 not significant for massaged infants, $p=0.117$, Cohen's $d=$ 0.76; factor time within control, IGF-1 levels at T1 vs T2 significant for control infants, $p<0.001$, Cohen's $d=2.97$; mean IGF-1 variation between $\mathrm{T} 1$ and $\mathrm{T} 2$ in control infants, $-0.650 \pm 0.08$, significantly higher than in massaged infants, $-0.183 \pm 0.03$, Cohen's $d=3.625$, $t$ test, $p<0.001$ ]. This results in significantly higher levels of circulating IGF-1 in massaged infants at T2 [twoway repeated measures ANOVA, treatment $(\mathrm{df}=1) \times$ time $(\mathrm{df}=$ $\operatorname{IGFBP3}(p=0.1)$. b
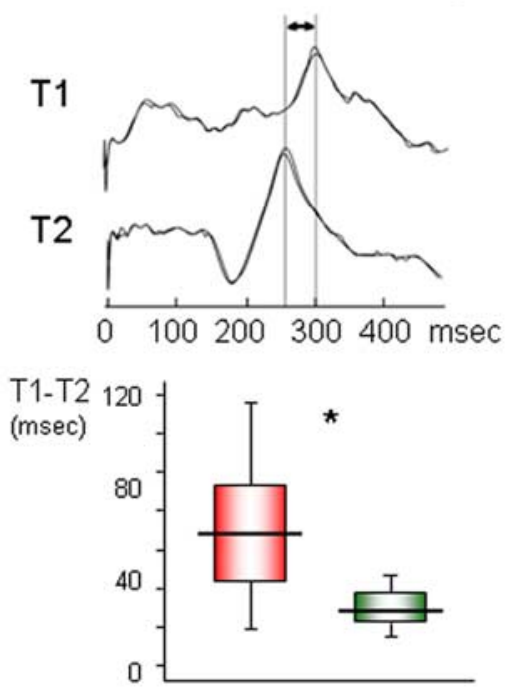

d

IGF1 path and Cortisol (T2-T1)

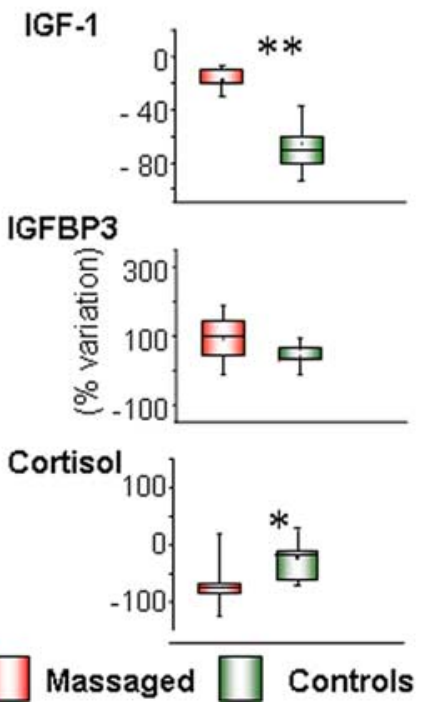

Figure 1. $\quad \boldsymbol{a}$, Difference between premassage and postmassage assessment of maximum interburst interval (EEG). Boxes indicate median (black horizontal line), interquartile values, and range. Massaged infants show larger differences between 22 and $\mathrm{T} 1$, which are statistically significant from those in controls (Mann-Whitney test, ${ }^{*} p=0.011$ ). $\boldsymbol{b}$, Difference between premassage and postmassage assessment of N300 latency (fVEP). Massaged infants show larger differences between T2 and T1, which are statistically significant from those in controls (Mann-Whitney test, ${ }^{*} p=0.013$ ). $c$, Behavioral visual acuity measured in cycles per degree (c/deg) by means of the Vital-Durand Acuity Cards at 3 and 7 months corrected age. Bars indicate mean values and SEM. Visual acuity in massaged infants is significantly higher than in controls at 3 months ( ${ }^{*} p=0.001, t$ test). This difference is no longer present at 7 months. Normal values for term-born infants are $3-5.2 \mathrm{c} /{ }^{\circ}$ at 3 months and $6.8-8.8$ at 6 months.d, Variation between premassage and postmassage assessment of blood IGF1, IGFBP3, and cortisol (T2 - T1/T1). Differences between the two experimental groups are significant for IGF-1, with massaged infants showing a smaller decrease between T1 and T2 $\left({ }^{* *} p=\right.$ $0.001)$, and for cortisol, with massaged infants showing a stronger decrease $\left({ }^{*} p=0.029\right)$. No significant difference was found for

1), pairwise multiple comparison procedure Holm-Sidak, factor treatment within T2, massage vs control significant, $p=0.016]$. The median values of IGF-1 blood levels at T2 is $81 \mathrm{ng} / \mathrm{ml}$ for massaged infants $(\mathrm{IQ}=[74,93])$ and $33 \mathrm{ng} / \mathrm{ml}$ for control infants $(\mathrm{IQ}=[28,41])($ Cohen's $d=2.14)$. This suggests an effect of the massage therapy in maintaining higher blood levels of IGF-1 during this period of development. 
Table 1. Results obtained in two monozygotic twins

\begin{tabular}{llc}
\hline Control & Monozygotic twins & Massaged \\
\hline-26.8 & Cortisol $\Delta(\mathrm{ng} / \mathrm{ml})$ & -72 \\
-98 & IGF1 $\Delta(\mathrm{ng} / \mathrm{ml})$ & -27.9 \\
+0.5 & IGFBP3 $\Delta(\mathrm{mg} / \mathrm{ml})$ & +0.9 \\
+17.8 & Weight $\Delta(\mathrm{g} / \mathrm{d})$ & +18.3 \\
-2 & EEG max interburst interval $\Delta(\mathrm{s})$ & -6 \\
-26.2 & VEP N300 $\Delta(\mathrm{ms})$ & -58.8 \\
4.8 & Visual acuity at 3 months $\left(\mathrm{c} /{ }^{\circ}\right)$ & 6.5 \\
7.9 & Visual acuity at 9 months $\left(\mathrm{c} /{ }^{\circ}\right)$ & 8.1 \\
\hline
\end{tabular}

$\Delta$ is the variation of the measured variable between the first assessment (T1) and the second assessment (T2). max, Maximum.

IGFBP-3 levels increase between the first and the second assessment for both groups, but only for massaged infants were IGFBP3 levels at the second assessment significantly different from those at the first one $(n=7$ for both groups, two-way repeated measures ANOVA, pairwise multiple comparison procedure Holm-Sidak, factor time within massage, IGFBP-3 levels at T1 vs T2 for massaged infants significant, $p=0.01$, Cohen's $d=1.32$; factor time within control, IGFBP-3 levels at T1 vs T2 not significant, $p=0.076$, Cohen's $d=0.79$ ). The direct comparison of the amount of IGFBP3 increase between second and first assessment in massaged versus control group but did not reach significance $(t$ test, $p=0.15$; mean \pm SEM IGFBP3 increase, $0.889 \pm 0.27$ in massaged infants and $0.4 \pm 0.15$ in control infants) (Fig. 1d).

Data in the literature also report decreased cortisol levels in infants undergoing massage therapy (Schanberg and Field, 1987), although this result has been questioned (Vickers et al., 2004). We measured blood cortisol in our infants, finding a reduction between the first and the second assessment for both massaged and control infants, but cortisol levels at the second assessment differed significantly from those at the first assessment only in the former group (massaged infants, blood cortisol at T1 differs from blood cortisol at T2, paired $t$ test, $p=0.015$, Cohen's $d=1.845$; control infants, blood cortisol at T1 does not differ from blood cortisol at T2, paired $t$ test, $p=0.09$, Cohen's $d=0.84, n=8$ for both groups). The amount of cortisol reduction between first and second assessment was significantly higher in massaged than in control subjects (Fig. 1d) (Mann-Whitney rank sum test, $p=$ $0.029, n=9$ for both groups, median and interquartiles for massage infants $-0.8,[-0.81,-0.48]$, median and interquartiles for control infants $-0.178[-0.526 .-0.13])$.

\section{Tactile stimulation accelerates visual system development in rat pups}

The results obtained in the human infant prompted us to explore the effect of tactile stimulation on visual development in the rat to unravel the possible underlying cellular and molecular mechanisms. Artificial manipulation and tactile stimulation in pups can influence eye opening in rodents (Smart et al., 1990) and regulates the expression of hormones important for development (Kuhn and Schanberg, 1998; Schanberg et al., 2003). The effect of tactile stimulation on visual development is, however, totally unknown.

Three daily tactile stimulation sessions of $15 \mathrm{~min}$ each were performed in newborn rats from P1 to P12.

As for human infants, we first assessed the developmental time course of the response to a flash by means of visual evoked potentials recorded from the primary visual cortex (fVEP). Recordings were performed between P14 and P18, when the maximum degree of latency shortening is normally observed. In line with the results in human subjects, we found a significantly faster rate of reduction of latencies in the massaged rats with respect to control rats. A significant difference in the two groups was found up to P16 (Fig. 2a) [two-way ANOVA, age $(\mathrm{df}=4) \times$ treatment $(\mathrm{df}=$ 1 ), factor age significant, $p<0.001(F=8.269)$, factor treatment significant, $p<0.001(F=31.984)$, pairwise multiple comparison procedure Holm-Sidak, treatment within P14-P16, massage vs controls, $p<0.05$; treatment within P17 and P18, massage vs controls, $p>0.05, n=4$ for each experimental group at each age].

To assess the effects of tactile stimulation on visual acuity development, we measured visual acuity at P25 by means of VEP recordings from the primary visual cortex. P25 is the age at which $\mathrm{EE}$ animals start to differ from non-EE animals (Cancedda et al., 2004; Landi et al., 2007). Electrophysiological visual acuity was significantly higher in the massaged rats $(n=11$; mean \pm SEM, $\left.0.743 \pm 0.02 \mathrm{c}^{\circ}\right)$ with respect to control rats $(n=7$; mean visual acuity, $0.63 \pm 0.03 \mathrm{c}^{\circ}$ ) (Fig. $2 b$ ). To rule out the possibility that these effects were attributable to the separation from the mother, a third experimental group was introduced, consisting of rats that were separated from the mother for the same amount of time as the massaged rats but receiving no tactile stimulation (maternal separation group, $n=12$ ). No differences were found between this latter group (mean visual acuity, $0.66 \pm 0.02 \mathrm{c}^{\circ}$ ) and the controls, supporting the role of tactile stimulation per se in producing the observed effects on visual acuity development [oneway ANOVA, factor treatment $(\mathrm{df}=2)$ significant, $p=0.003$ $(F=7.38)$, multiple comparison procedure Holm-Sidak method, visual acuity of massage rats differs from that of control rats and of maternal separation rats, Cohen's $d=1.827$ and 1.64, respectively; the latter two do not differ, Cohen's $d=0.64]$.

To assess whether the effects of tactile stimulation on pup visual acuity were evident also at behavioral level, we measured visual acuity by means of the Prusky water box at P25 and P28. We found that visual acuity in massage rats $(n=15)$ was significantly higher than in controls $(n=13)$ and in maternal separation rats $(n=11)$ at both P25 (mean \pm SEM visual acuity, $0.674 \pm 0.009 \mathrm{c} /{ }^{\circ}$ in massage, $0.6 \pm 0.01 \mathrm{c} /{ }^{\circ}$ in maternal separation, $0.59 \pm 0.01 \mathrm{c} /{ }^{\circ}$ in controls) and at P28 (mean \pm SEM visual acuity, $0.76 \pm 0.009 \mathrm{c} /{ }^{\circ}$ in massage, $0.674 \pm 0.014 \mathrm{c} /{ }^{\circ}$ in maternal separation, $0.65 \pm 0.013 \mathrm{c} /{ }^{\circ}$ in controls); visual acuity in the latter groups did not differ [two-way repeated measures ANOVA, treatment $(\mathrm{df}=2) \times$ age $(\mathrm{df}=1)$, factor age significant, $p<$ $0.001(F=48.86)$, factor treatment significant, $p<0.001(F=$ $11.4)$, visual acuity in massage group different from controls and maternal separation groups at both ages, $p<0.05$; multiple comparison procedure Holm-Sidak method].

The fact that the effects of massage on visual acuity are apparent at both behavioral and electrophysiological level rules out the possibility that the effects on behavior are simply attributable to aspecific effects of massage on pup attention or motivation and that the effects on visual cortex physiology are devoid of any behavioral relevance.

As found in massaged infants, a higher visual acuity in rats subjected to tactile stimulation was evident at an early stage of visual acuity development but was no longer found at older ages (P45; data not shown).

To compare the effects of massage on visual acuity development with those of exposure to environmental enrichment, we exposed another group of newborn rats to a time-restricted protocol of EE for a period of $12 \mathrm{~d}[\mathrm{P} 0-\mathrm{P} 12$, the period during which our massage protocol was applied, which is also the time during which higher levels of maternal stimulation have been reported in 


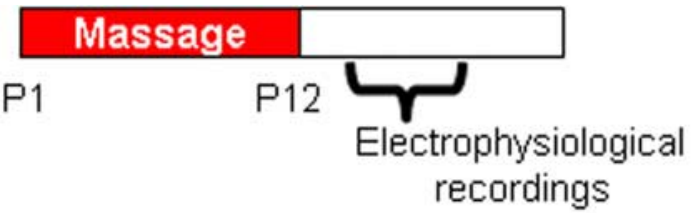

\section{Massage}

P12

P25-P28

Assessment of

visual acuity
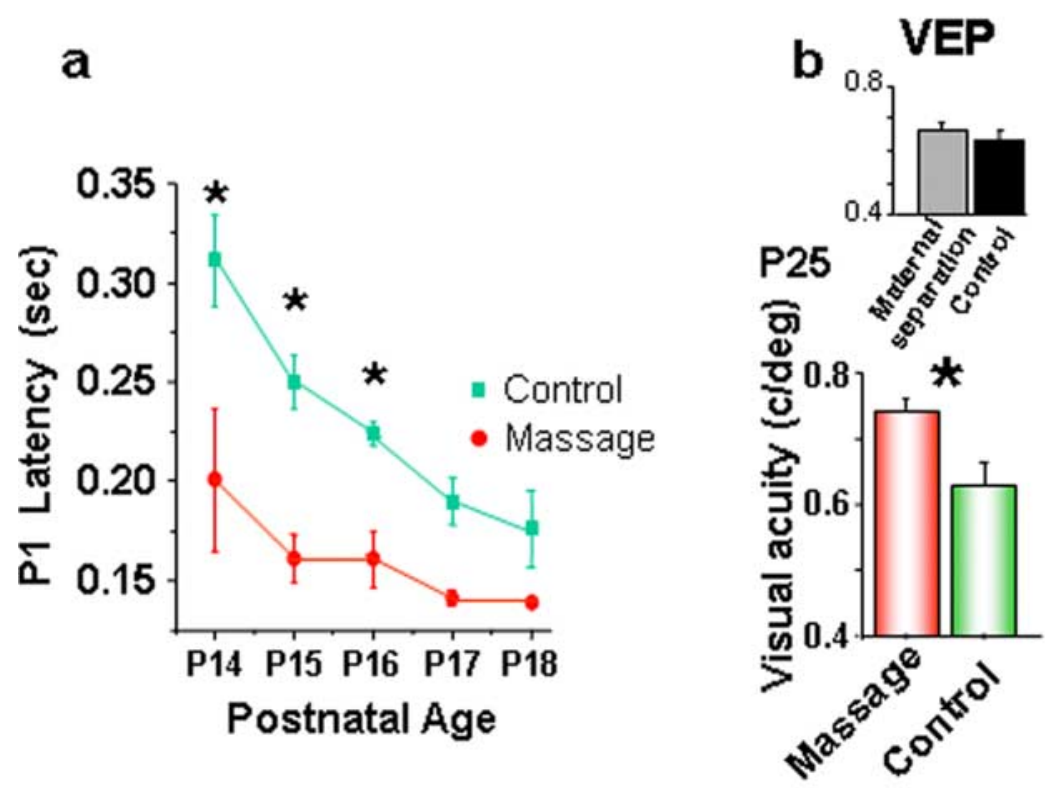

\section{Behavior}

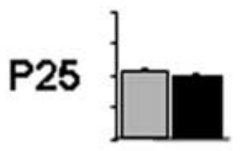

P28
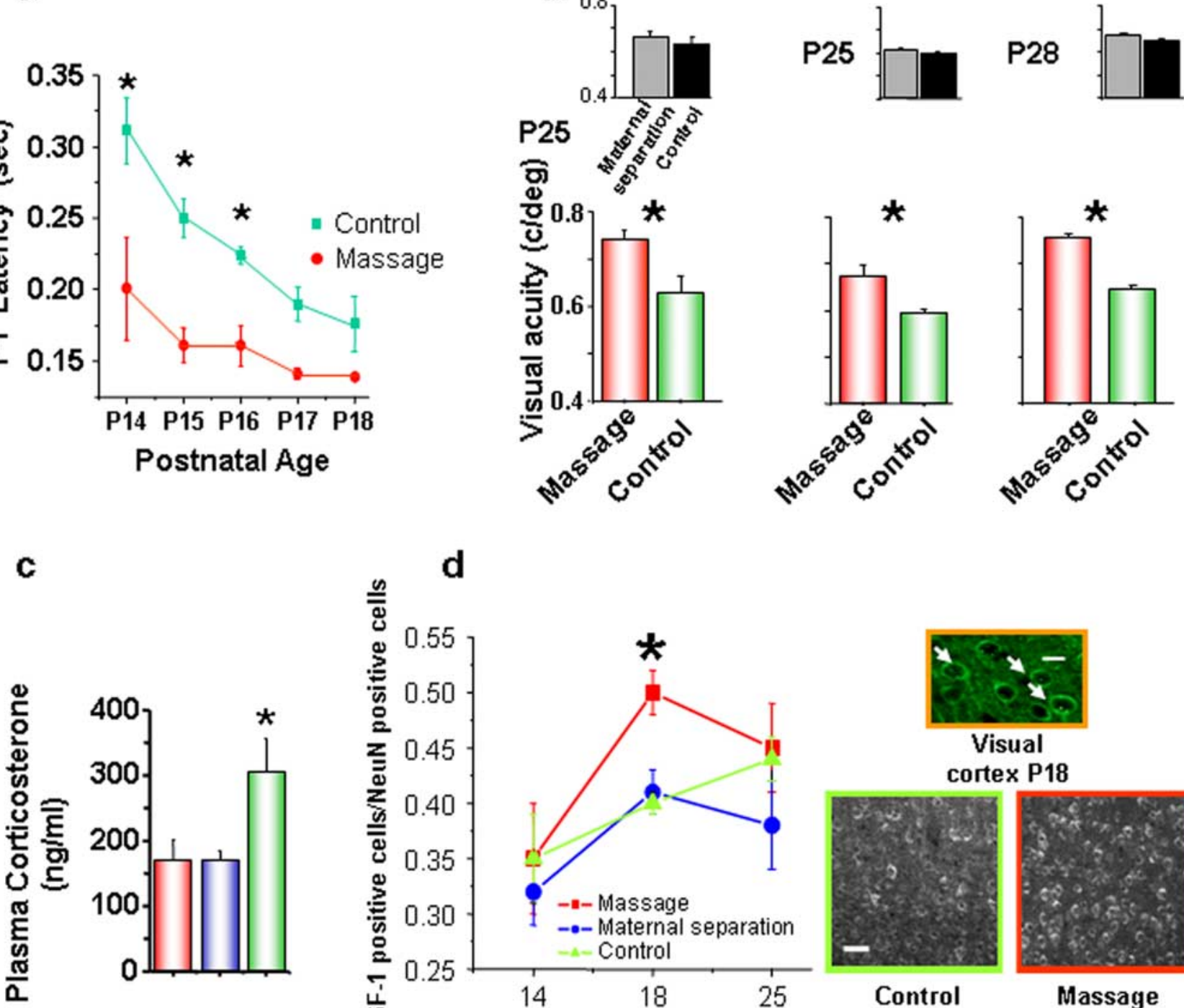

$\square$ Control $\square$ Massage $\square$ Maternal
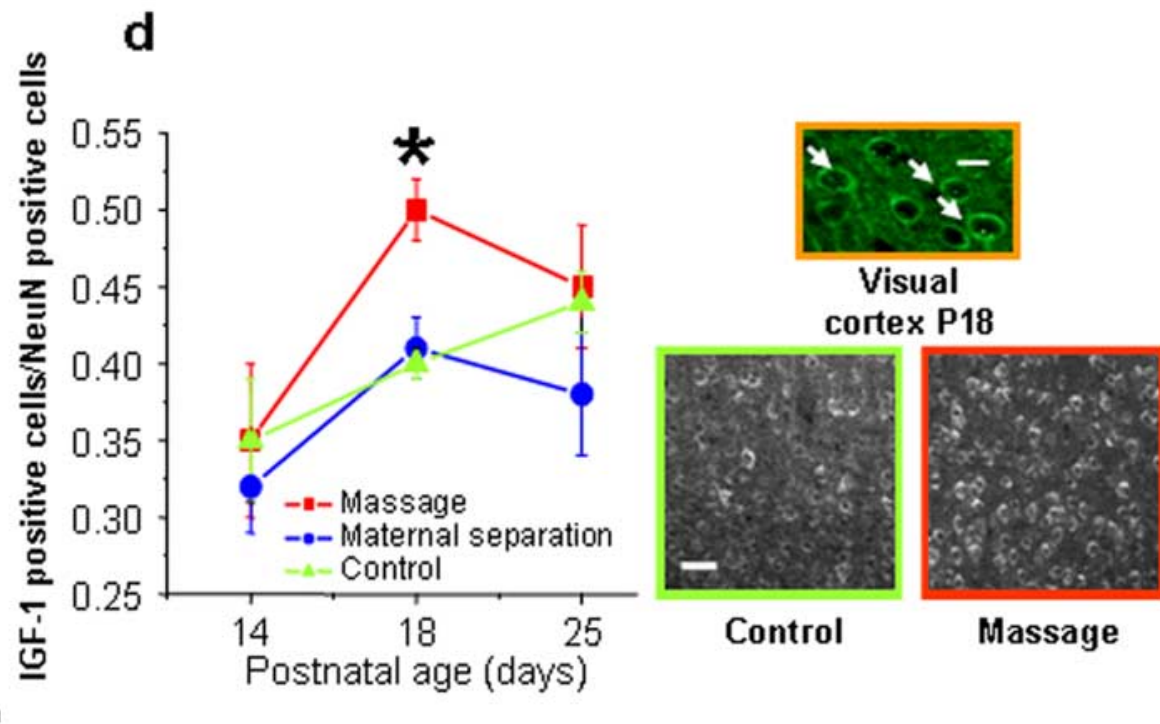

Figure 2. $\boldsymbol{a}$, Massage in rat pups determines a significantly faster rate of reduction in the latencies of the flash VEP. Top, Experimental protocol. Bottom, Mean P1 latency plotted as a function of age for the groups of massaged and of control rats. The developmental shortening of $P 1$ latency is significantly faster for massaged rats than control rats (two-way ANOVA, treatment $\times$ age, factor age $p<0.001$, factor treatment $p<0.001, n=4$ for each age group both for massaged and controls, Holm-Sidak post hoc test). Asterisks denote significant difference between treatments at a given postnatal day. Vertical bars represent SEM. $\boldsymbol{b}$, Massage in rat pups affects visual acuity development. Top, Experimental protocol. Bottom left, Mean visual acuity determined at $\mathrm{P} 25$ by means of VEPs recorded from the primary visual cortex for massaged rats $(n=11)$ and for control rats $(n=7)$. Vertical bars are SEM. Massage group significantly differs from control group and from maternal separation group ( $n=12$; inset) (one-way ANOVA, factor treatment significant, $p=0.003$, multiple comparison procedure Holm-Sidak method, visual acuity of massage rats differs from that of control rats and of maternal separation rats; the latter two do not differ). Bottom right, Mean behavioral visual acuity in P25 and P28 massage, controls, and maternal separation groups. Vertical bars represent SEM. Visual acuity of massaged rats $(n=15)$ is significantly higher than in control $(n=13)$ or in maternal separation $(n=11$; inset) (two-way repeated measures ANOVA, treatment $\times$ age, factor age significant, $p<0.001$, factor treatment significant, $p<0.001$, visual acuity in massage group different from controls and maternal separation groups at both ages, $p<$ 0.05 ; visual acuity in controls and maternal separation group not significantly different, multiple comparison procedure Holm-Sidak method). Asterisks denote significant difference. $c /$ deg, $c /{ }^{\circ}$. $\boldsymbol{c}$, Mean plasma corticosterone levels at P14 in massage, maternal separation, and control groups. The level of corticosterone in the control groups is significantly higher than in the maternal separation and massage groups (one-way ANOVA, $p<0.02, n=6$ for each group, post hoc Tukey's test). Vertical bars denote SEM. Asterisks denote significant difference. $\boldsymbol{d}$, Left, Mean number of IGF-1-positive cells in the visual cortex, normalized to the number of neurons (NeuN-positive cells) for each developmental age analyzed. $n=4$ for each age and each experimental group. Vertical bars represent SEM. The normalized number of IGF-1-p ositive cells increases significantly between P15 and P18; the normalized number at P18 in massaged rats is significantly increased with respect to maternal separation and control rats (two-way ANOVA, treatment $\times$ age, factor treatment $p=0.024$, factor age $p<0.01$, post hoc Tukey's test). Top right, Typical appearance of IGF-1-positive cells in the developing rat binocular visual cortex (P25). Scale bar, $25 \mu \mathrm{m}$. Bottom right, Examples of IGF-1 labeling at P18 for one massaged and one control rat. Scale bar, $50 \mu \mathrm{m}$. 
EE pups compared with controls (Sale et al., 2004)] and assessed visual acuity at P25 by means of VEP. We found that the effects of EE up to P12 on visual acuity development observed at P25 were not significantly different from those in massaged rats, both being less marked than those observed previously in P25 animals exposed to EE up to P25 (Cancedda et al., 2004; Sale et al., 2004; Landi et al., 2007) [mean \pm SEM visual acuity for P25 rats EE up to $\mathrm{P} 12,0.74 \pm 0.01 \mathrm{c} /{ }^{\circ}, n=5$, difference with control $\mathrm{P} 25$ rats $(n=7)$ significant, difference with massaged rats $(n=11)$ nonsignificant, difference with rats EE up to $\mathrm{P} 25\left(0.93 \pm 0.03 \mathrm{c} /{ }^{\circ} ; n=\right.$ 5) significant, one-way ANOVA ( $\mathrm{df}=3), p<0.001, F=33,558$, multiple comparison procedure Holm-Sidak method)]. We thus conclude that tactile stimulation provided by maternal care is likely to be the main factor responsible for the effects on visual development of early exposure to EE, when pup interactions with the external environment are mainly represented by their interactions with the mother.

\section{The acceleration of visual system development in massaged rat pups is not attributable to a modulation of stress hormone levels}

Tactile stimulation in rodent pups reduces the increase in corticosterone caused by stressful events (Pauk et al., 1986). To control whether a modulation of stress hormone levels contributes to the documented effects on visual acuity development, we measured plasma corticosterone levels in P14 rats subjected to the protocol of stimulation, subjected to separation, or left undisturbed. We found (Fig. $2 c$ ) that corticosterone levels were significantly lower than in controls $(n=6$; mean \pm SEM, $306 \pm 50$ $\mathrm{ng} / \mathrm{ml}$ ) for both massaged $(n=6$; mean \pm SEM, $171 \pm 30 \mathrm{ng} / \mathrm{ml})$ and separated pups (mean \pm SEM, $171 \pm 12 \mathrm{ng} / \mathrm{ml}$ ), with no difference between themselves [one-way ANOVA $(\mathrm{df}=2), F=$ $5.147, p=0.02$, post hoc Tukey's test)]. Because only in massaged rats there was a significant effect on visual acuity development, we conclude that modulation of stress hormones does not contribute to tactile stimulation effects on visual development.

Increased levels of IGF-1 in the cortex of massaged rat pups It is known that EE and physical exercise increase IGF-1 in the brain (Carro et al., 2000, 2001; Koopmans et al., 2006; Ciucci et al., 2007; Sale et al., 2007).

We assessed in massaged animals whether IGF-1 levels were increased in the brain and in particular in the primary visual cortex and in another sensory cortex, area Te1, the primary auditory cortex. In both areas, we quantified the presence of IGF-1 in terms of the number of IGF-1-positive cells normalized to the number of neurons (NeuN-positive cells) (Ciucci et al., 2007).

In the primary visual cortex, IGF-1 presence was analyzed at P14, P18, and P25, as in the study by Ciucci et al. (2007). At P14 and P25, no difference was found between massaged, separated, and control rats (Fig. $2 d$ ). At P18, the presence of IGF-1 in the visual cortex of massaged rats was increased with respect to P18 separated or control rats; the latter two did not differ between themselves (Fig. $2 d$ ) [two-way ANOVA, treatment $(\mathrm{df}=2) \times$ age $(\mathrm{df}=2)$, factor treatment $p=0.024(F=4.2)$, factor age $p<$ $0.001(F=9.27)$, post hoc Tukey's test]. Thus, massage increases IGF-1 in the visual cortex.

To control whether the effects of massage in IGF-1 brain levels were specific for visual areas, we assessed the presence of IGF-1 in the primary auditory cortex at P14. We found that IGF-1 content was significantly increased in massage pups $(n=5)$ with respect to controls $(n=3)$ also in the auditory cortex (mean \pm SEM
IGF-1-positive cells/NeuN-positive cells in massage pups, $0.52 \pm$ 0.022; in control pups, $0.43 \pm 0.02$; $t$ test, $p=0.04$ ).

Thus, the increase in IGF-1 in massage pups is not specific for the visual cortex. This is not surprising because it is known that early exposure to EE increases IGF-1 levels very precociously in the retina (up to P10) and in the cerebellum (P1) of EE pups and higher levels of IGF-1 are found in the milk (Sale et al., 2007), supporting the idea that EE and massage act on brain development, not on development of specific brain regions.

We have demonstrated recently that blocking IGF-1 action in the visual cortex from P18 to P25 blocked EE effects on rat visual acuity development (Ciucci et al., 2007). We assessed now whether blocking IGF-1 action more precociously and systemically blocks the effects of massage. We used the IGF-1 antagonist JB1, already used to antagonize circulating IGF-1 action (Carro et al., 2001) and, locally infused, to block IGF-1 action in the visual cortex (Ciucci et al., 2007).

We investigated whether blocking IGF-1 action by means of systemic JB1 injections from P1 to P10 would block the effects of massage on visual acuity development. JB1 injections were performed in massaged rats (massage plus JB1 rats) subcutaneously every other day. Visual acuity was assessed electrophysiologically (VEPs) at P25. We found that, in massaged plus JB1 rats $(n=8)$, visual acuity $\left(0.61 \pm 0.03 \mathrm{c}^{\circ}\right)$ did not differ from that in control, nonmassaged rats and was significantly lower than in massaged rats [one-way ANOVA ( $\mathrm{df}=3), p<0.001(F=7.907)$ post hoc Holm-Sidak method, massaged vs massaged plus JB1 $p<0.05$; control vs massaged, $p<0.05$; control vs massaged plus JB1 $p>$ 0.05]. As control for aspecific effects of injections, another group of massaged rat pups was injected with saline with the same procedure used to inject JB1. No difference between the visual acuity of these rats ( $n=4$; massaged plus saline; visual acuity, $0.72 \pm$ $\left.0.02 \mathrm{c}^{\circ}\right)$, and the visual acuity for massaged rats was evident [one-way ANOVA $(\mathrm{df}=3) p<0.001(F=7.907)$ post hoc HolmSidak method, massaged plus saline vs massaged rats $p>0.05$, massaged plus JB1 vs massaged plus saline $p<0.05$ ].

\section{Discussion}

Our data indicate that enriching the environment in terms of body massage and multisensory stimulation ("massage therapy") affects brain development and in particular visual system maturation in both human and rat pups. This not only underlines the importance of the environment as a driving force in early postnatal development but suggests that the environment acts by modulating the level of endogenous factors such as IGF-1, which regulate brain growth, and in particular, the development of visual cortex.

The effects of massage on visual acuity development were evident at 3 months of age in infants, that is more than 2 months after the end of the massage protocol, but were no longer present at 7 months of age. At this stage, we cannot say whether this is attributable to a greater sensitivity of the earliest phase of visual acuity development, characterized by the most rapid maturation of the visual cortex and of visual acuity, to the endogenous factors modulated by massage or to the rather short period of massage protocol used. It would also be of interest to study the effects of massage on visual acuity development in infants born at term and compare the results with those obtained in our rat pups who were born at term.

The effects of massage in accelerating visual development and in increasing IGF-1 levels in rat pups are not attributable to the pup manipulation or to the simple removal from the nest or to modulation of stress hormone levels, because they are absent in 
pups separated from the mother for the same amount of time as massaged pups but not subjected to massage. This supports the importance of massage per se in promoting brain development. These results also support the hypothesis that the level of tactile stimulation provided by licking/grooming is an important regulator of brain development (Liu et al., 2000; Weaver et al., 2004, 2006, 2007; Meaney and Szyf, 2005).

This is the first time that body massage is shown to affect brain development in human infants, and, in particular, it is the first time that tactile stimulation is shown to influence visual development. The studies previously conducted in infants using massage therapy assessed body weight, levels of stress hormones such as cortisol, growth hormone (Schanberg and Field, 1987; Vickers et al., 2004), and IGF-1 (Field et al., 2008), but did not investigate massage effects on brain development.

Our results suggest that massage effects are not specific for the visual system. Indeed, we find changes in the maturation of cerebral electrical activity in infants that are evident at all EEG electrodes, not only at the occipital ones. In addition, IGF-1 immunoreactivity changes in rats are found not only in the visual cortex but also in the auditory cortex. This is in accordance with data obtained in rats precociously exposed to environmental enrichment, which receive a higher level of tactile stimulation through licking and grooming during the early phases of their postnatal development (Sale et al., 2004); in these animals, increases in IGF-1 levels are found not only in the visual cortex but also in the retina and in the cerebellum (Ciucci et al., 2007; Sale et al., 2007). All these results support the idea that both $\mathrm{EE}$ and massage act on brain development, not on development of specific brain regions.

\section{Which mechanism could underlie the effects of massage on visual development?}

We have shown that massage increases blood levels of IGF-1 in infants. In addition, the results in rats show that massage is effective in increasing IGF-1 levels in the cortex and in particular in the visual cortex at P18. Such an increase could promote the changes necessary to accelerate visual acuity development. We have indeed recently shown that exogenous administration of IGF-1 from P18 to P25 in the rat visual cortex is sufficient to accelerate visual acuity development, mimicking EE effects, and that blocking IGF-1 action in the visual cortex of rats exposed to EE prevents the acceleration of visual acuity development (Ciucci et al., 2007). In this study, we found that blocking IGF-1 action blocks the effects of massage, showing that IGF-1 is also crucial for the effects of massage on rat visual acuity development.

This strongly suggests that IGF-1 is one of the mediators of massage therapy effects on visual development also in infants.

The action of an increase in IGF-1 on visual acuity development is likely to be mediated by the accelerated maturation of intracortical inhibitory circuitry (Ciucci et al., 2007); the development of visual acuity is correlated with a decrease in cortical receptive field size (Fagiolini et al., 1994) and with postnatal development of intracortical inhibitory circuits that shape visual cortical receptive fields (Sillito, 1975; Hensch et al., 1998). Indeed, also the accelerated development of visual acuity in EE mice is accompanied by a precocious increase in the expression of GABA biosynthetic enzymes, GAD65/67, in the visual cortex. In line with this, visual acuity development is faster in BDNFoverexpressing mice that show an accelerated development of inhibition in the visual cortex (Huang et al., 1999).

Recently, lower levels of plasma IGF-1 and IGFBP3 in premature infants have been correlated with a higher incidence of ROP (Hellström et al., 2003; Löfqvist et al., 2006, 2007). Because mas- sage therapy increases levels of plasma IGF-1 and, to a lower extent, of plasma IGFBP3 in infants, it could have important clinical applications particularly in preterm infants between postmenstrual age weeks 30-35, when induction of ROP occurs (Hellström et al., 2003).

\section{References}

Als H, Lawhon G, Duffy FH, McAnulty GB, Gibes-Grossman R, Blickman JG (1994) Individualized developmental care for the very low-birth-weight preterm infant: medical and neurofunctional effects. JAMA 272:853-858.

Biagioni E, Bartalena L, Boldrini A, Cioni G, Giancola S, Ipata AE (1994) Background EEG activity in preterm infants: correlation of outcome with selected maturational features. Electroencephalogr Clin Neurophysiol 91:154-162.

Biagioni E, Bartalena L, Biver P, Pieri R, Cioni G (1996) Electroencephalographic dysmaturity in preterm infants: a prognostic tool in the early postnatal period. Neuropediatrics 27:311-316.

Burton CL, Chatterjee D, Chatterjee-Chakraborty M, Lovic V, Grella SL, Steiner M, Fleming AS (2007) Prenatal restraint stress and motherless rearing disrupts expression of plasticity markers and stress-induced corticosterone release in adult female Sprague-Dawley rats. Brain Res 1158:28-38.

Cancedda L, Putignano E, Sale A, Viegi A, Berardi N, Maffei L (2004) Acceleration of visual system development by environmental enrichment. J Neurosci 24:4840-4848.

Carro E, Nuñez A, Busiguina S, Torres-Aleman I (2000) Circulating insulinlike growth factor I mediates effects of exercise on the brain. J Neurosci 20:2926-2933.

Carro E, Trejo JL, Busiguina S, Torres-Aleman I (2001) Circulating insulinlike growth factor I mediates the protective effects of physical exercise against brain insults of different etiology and anatomy. J Neurosci 21:5678-5684.

Champagne FA, Curley JP (2009) Epigenetic mechanisms mediating the long-term effects of maternal care on development. Neurosci Biobehav Rev 33:593-600.

Chatterjee D, Chatterjee-Chakraborty M, Rees S, Cauchi J, de Medeiros CB, Fleming AS (2007) Maternal isolation alters the expression of neural proteins during development: "stroking" stimulation reverses these effects. Brain Res 1158:11-27.

Ciucci F, Putignano E, Baroncelli L, Landi S, Berardi N, Maffei L (2007) Insulin-like growth factor 1 (IGF-1) mediates the effects of enriched environment (EE) on visual cortical development. PLoS ONE 2:e475.

Fagiolini M, Pizzorusso T, Berardi N, Domenici L, Maffei L (1994) Functional postnatal development of the rat primary visual cortex and the role of visual experience: dark rearing and monocular deprivation. Vision Res 34:709-720.

Fernandez AM, Gonzalez de la Vega AG, Planas B, Torres-Aleman I (1999) Neuroprotective actions of peripherally administered insulin-like growth factor I in the injured olivo-cerebellar pathway. Eur J Neurosci 11:2019-2030.

Field T, Diego M, Hernandez-Reif M, Dieter JN, Kumar AM, Schanberg S, Kuhn C (2008) Insulin and insulin-like growth factor-1 increased in preterm neonates following massage therapy. J Dev Behav Pediatr 29:463-466.

Hellström A, Engström E, Hård AL, Albertsson-Wikland K, Carlsson B, Niklasson A, Löfqvist C, Svensson E, Holm S, Ewald U, Holmström G, Smith LE (2003) Postnatal serum insulin-like growth factor I deficiency is associated with retinopathy of prematurity and other complications of premature birth. Pediatrics 112:1016-1020.

Hensch TK, Fagiolini M, Mataga N, Stryker MP, Baekkeskov S, Kash SF (1998) Local GABA circuit control of experience-dependent plasticity in developing visual cortex. Science 282:1504-1508.

Huang ZJ, Kirkwood A, Pizzorusso T, Porciatti V, Morales B, Bear MF, Maffei L, Tonegawa S (1999) BDNF regulates the maturation of inhibition and the critical period of plasticity in mouse visual cortex. Cell 98:739-755.

Koopmans GC, Brans M, Gómez-Pinilla F, Duis S, Gispen WH, TorresAleman I, Joosten EA, Hamers FP (2006) Circulating insulin-like growth factor I and functional recovery from spinal cord injury under enriched housing conditions. Eur J Neurosci 23:1035-1046.

Kuhn CM, Schanberg SM (1998) Responses to maternal separation: mechanisms and mediators. Int J Dev Neurosci 16:261-270.

Landi S, Sale A, Berardi N, Viegi A, Maffei L, Cenni MC (2007) Retinal 
functional development is sensitive to environmental enrichment: a role for BDNF. FASEB J 21:130-139.

Liu D, Diorio J, Day JC, Francis DD, Meaney MJ (2000) Maternal care, hippocampal synaptogenesis and cognitive development in rats. Nat Neurosci 3:799-806.

Löfqvist C, Andersson E, Sigurdsson J, Engström E, Hård AL, Niklasson A, Smith LE, Hellström A (2006) Longitudinal postnatal weight and insulin-like growth factor I measurements in the prediction of retinopathy of prematurity. Arch Ophthalmol 124:1711-1718.

Löfquist C, Chen J, Connor KM, Smith AC, Aderman CM, Liu N, Pintar JE, Ludwig T, Hellstrom A, Smith LE (2007) IGFBP3 suppresses retinopathy through suppression of oxygen-induced vessel loss and promotion of vascular regrowth. Proc Natl Acad Sci U S A 104:10589-10594.

Meaney MJ, Szyf M (2005) Environmental programming of stress responses through DNA methylation: life at the interface between a dynamic environment and a fixed genome. Dialogues Clin Neurosci 7:103-123.

Nithianantharajah J, Hannan AJ (2006) Enriched environments, experience-dependent plasticity and disorders of the nervous system. Nat Rev Neurosci 7:697-709.

Pauk J, Kuhn CM, Field TM, Schanberg SM (1986) Positive effects of tactile versus kinesthetic or vestibular stimulation on neuroendocrine and ODC activity in maternally-deprived rat pups. Life Sci 39:2081-2087.

Prusky GT, West PW, Douglas RM (2000) Behavioral assessment of visual acuity in mice and rats. Vision Res 40:2201-2209.

Rosenzweig MR, Bennett EL, Hebert M, Morimoto H (1978) Social grouping cannot account for cerebral effects of enriched environments. Brain Res 153:563-576.

Sale A, Putignano E, Cancedda L, Landi S, Cirulli F, Berardi N, Maffei L (2004) Enriched environment and acceleration of visual system development. Neuropharmacology 47:649-660.

Sale A, Cenni MC, Ciucci F, Putignano E, Chierzi S, Maffei L (2007) Maternal enrichment during pregnancy accelerates retinal development of the fetus. PLoS ONE 2:e1160.

Schanberg SM, Field TM (1987) Sensory deprivation stress and supplemental stimulation in the rat pup and preterm human neonate. Child Dev 58:1431-1447.

Schanberg SM, Ingledue VF, Lee JY, Hannun YA, Bartolome JV (2003) PKC alpha mediates maternal touch regulation of growth-related gene expression in infant rats. Neuropsychopharmacology 28:1026-1030.

Scher MS, Steppe DA, Dokianakis SG, Sun M, Guthrie RD, Sclabassi RJ (1994) Cardiorespiratory behavior during sleep in full-term and preterm neonates at comparable postconceptional term ages. Pediatr Res $36: 738-744$.
Sillito AM (1975) The contribution of inhibitory mechanisms to the receptive field properties of neurones in the striate cortex of the cat. J Physiol 250:305-329.

Smart JL, McMahon AC, Massey RF, Akbar GN, Warren MA (1990) Evidence of non-maternally mediated acceleration of eye-opening in "enriched" artificially reared rat pups. Brain Res Dev Brain Res 56:141-143.

Stockard-Pope JE, Werner SS, Bickford RG (1992) Atlas of neonatal electroencephalography, Ed 2 (Werner SS, ed). New York: Raven.

Symington A, Pinelli J (2006) Developmental care for promoting development and preventing morbidity in preterm infants. Cochrane Database Syst Rev 2006:CD001814.

Teller DY, McDonald MA, Preston K, Sebris SL, Dobson V (1986) Assessment of visual acuity in infants and children: the acuity card procedure. Dev Med Child Neurol 28:779-789.

Tropea D, Kreiman G, Lyckman A, Mukherjee S, Yu H, Horng S, Sur M (2006) Gene expression changes and molecular pathways mediating activity-dependent plasticity in visual cortex. Nat Neurosci 9:660-668.

Tsuneishi S, Casaer P, Fock JM, Hirano S (1995) Establishment of normal values for flash visual evoked potentials (VEPs) in preterm infants: a longitudinal study with special reference to two components of the $\mathrm{N} 1$ wave. Electroencephalogr Clin Neurophysiol 96:291-299.

van Hof-van Duin J, Heersema DJ, Groenendaal F, Baerts W, Fetter WPF (1992) Visual field and grating acuity development in low-risk preterm infants during the first $2 \frac{1}{2}$ years after term. Behav Brain Res 49:115-122.

van Praag H, Kempermann G, Gage FH (2000) Neural consequences of environmental enrichment. Nat Rev Neurosci 1:191-198.

Vickers A, Ohlsson A, Lacy JB, Horsley A (2004) Massage for promoting growth and development of preterm and/or low birth-weight infants. Cochrane Database Syst Rev 2004:CD000390.

Vital-Durand F (1992) Acuity card procedures and the linearity of grating resolution development during the first year of human infants. Behav Brain Res 49:99-106.

Weaver IC, Cervoni N, Champagne FA, D’Alessio AC, Sharma S, Seckl JR, Dymov S, Szyf M, Meaney MJ (2004) Epigenetic programming by maternal behavior. Nat Neurosci 7:847-854.

Weaver IC, Meaney MJ, Szyf M (2006) Maternal care effects on the hippocampal transcriptome and anxiety-mediated behaviors in the offspring that are reversible in adulthood. Proc Natl Acad Sci U S A 103:3480-3485.

Weaver IC, D'Alessio AC, Brown SE, Hellstrom IC, Dymov S, Sharma S, Szyf M, Meaney MJ (2007) The transcription factor nerve growth factorinducible protein a mediates epigenetic programming: altering epigenetic marks by immediate-early genes. J Neurosci 27:1756-1768 . 